\title{
MENOPAUSAL HORMONE THERAPY DOES NOT INFLUENCE LUNG CANCER RISK: RESULTS FROM THE CALIFORNIA TEACHERS STUDY
}

\author{
Jessica Clague ${ }^{1,2}$, Peggy Reynolds ${ }^{3}$, Jane Sullivan-Halley ${ }^{1}$, Huiyan $\mathbf{M a}^{1}$, James V Lacey \\ Jr. ${ }^{1}$, Katherine D. Henderson ${ }^{1}$, Giske Ursin ${ }^{4,5}$, Dee West ${ }^{3}$, Shine Chang ${ }^{2}$, George L. \\ Delclos $^{6}$, Xianglin L. Du ${ }^{7}$, Michele R. Forman ${ }^{2}$, and Leslie Bernstein ${ }^{1}$ \\ ${ }^{1}$ Department of Population Sciences, Division of Cancer Etiology, Beckman Research Institute at \\ the City of Hope National Medical Center, Duarte, CA \\ ${ }^{2}$ Department Epidemiology, University of Texas, M. D. Anderson Cancer Center, Houston, TX \\ ${ }^{3}$ Cancer Prevention Institute of California, Fremont, California \\ ${ }^{4}$ Department of Preventive Medicine, Norris Comprehensive Cancer Center, University of \\ Southern California Keck School of Medicine, Los Angeles, California \\ ${ }^{5}$ Department of Nutrition, University of Oslo, Oslo, Norway \\ ${ }^{6}$ Department Environmental and Occupational Health Sciences, School of Public Health, \\ University of Texas Health Science Center, Houston, TX \\ ${ }^{7}$ Department Epidemiology, School of Public Health, University of Texas Health Science Center, \\ Houston, TX
}

\section{Abstract}

Background-Results from studies examining the association between hormone therapy (HT) and lung cancer risk disagree.

Methods-We examined the associations between HT use and lung cancer risk among 60,592 postmenopausal women enrolled in the prospective California Teachers Study cohort. Between 1995 and 2007, 727 women were diagnosed with lung cancer. Multivariable Cox proportional hazards regression models were fit using age as the time metric.

Results-No measure of HT use was associated with lung cancer risk (all p-values for trend $\geq 0.4$ ). In addition, no variations in risk by smoking status (never, ever, former, current), type of HT (E-alone, E+P use), type of menopause, or lung cancer histology were observed.

Conclusions-Our findings do not support an association between HT and lung cancer.

Impact-This large-scale, prospective study, which capitalizes on the detailed hormone use, smoking history, and type of menopause information available within this unique cohort, was unable to find any association between intake of HT and lung cancer risk.

${ }^{\dagger}$ Corresponding author: Leslie Bernstein, PhD, Professor and Director, Division of Cancer Etiology, Department of Population Sciences, Dean for Faculty Affairs, Beckman Research Institute, City of Hope National Medical Center, 1500 East Duarte Road, Duarte, CA 91010, (626) 471-7315, (626) 471-7308 (Fax), lbernstein@ coh.org. 


\section{Introduction}

Cigarette smoking, the leading cause of lung cancer, is responsible for $80-90 \%$ of lung malignancies(1). Nevertheless, lung cancers develop among lifetime never smokers highlighting the need to investigate other factors that may modify lung cancer risk(2). Lung cancer pathology, risk factors and prognosis differ by sex, prompting researchers to investigate hormonal and reproductive factors(3). The association between hormone therapy (HT) and lung cancer has been investigated; however, the results have differed across studies(4-5). We examined HT use in relation to lung cancer risk among women in the California Teacher Study (CTS) capitalizing on detailed information on HT use (including formulation, duration and timing), smoking history, and type of menopause.

\section{Methods}

\section{Study Population}

The CTS cohort, established in 1995-1996, consists of 133,479 active and retired female teachers and administrators(6). CTS participants residing in California are followed annually for cancer diagnosis and death. The questionnaire participants completed at baseline covered a wide range of issues related to cancer risk and women's health, including recent and past HT use, menopausal status, and cigarette smoking. For estrogen (E), questions were asked about Premarin ${ }^{\circledR}$ as well as other estrogens and information was collected on mode of administration, dose if Premarin ${ }^{\circledR}$ was used, ages at first and last use, and duration of use. For progestins $(\mathrm{P})$, participants were asked to report the type of progestin used, ages at first and last use, years of use, days per month of use, and dose. A five-category smoking variable was created based on baseline smoking status and median pack-years (never, former light, former heavy, current light, and current heavy).

Women were sequentially excluded from the analytic cohort if not a California resident at baseline $(\mathrm{n}=8,867)$, the medical history portion of the questionnaire was incomplete $(n=662)$, participant reported a prior diagnosis of lung cancer at baseline $(n=215)$, or participant limited her participation to breast cancer research $(n=18)$. Additionally, we excluded premenopausal women $(\mathrm{n}=47,976)$, women with indeterminate menopausal status $(\mathrm{n}=4,957)$, women missing information on smoking $(\mathrm{n}=2,924)$, and women with incomplete HT histories $(n=7,268)$. Of 60,592 eligible postmenopausal women, 727 were diagnosed with lung cancer a month or more after joining the cohort and before December 31, 2007.

\section{Statistical Analyses}

Relative risks (RR) and 95\% confidence intervals (CI) for lung cancer associated with HT use were estimated using multivariable Cox proportional hazards regression with age (days) used as the timescale. Models were age stratified (years) and adjusted for race/ethnicity, smoking status/pack-years, type of menopause, and body mass index (BMI, $\mathrm{kg} / \mathrm{m}^{2}$ ). Followup time began on the date the baseline questionnaire was completed and ended on the date of lung cancer diagnosis, death, move out of California, or December 31, 2007, whichever occurred first.

\section{Results}

A majority (74.5\%) of eligible postmenopausal women had used HT; $16.0 \%$ were former and $58.5 \%$ were current users of either E-alone or E+P therapy at baseline (Table 1). In the multivariable model, use of any HT was not associated with lung cancer risk (RR, 0.95, 95\% CI, 0.80-1.13) (Table 2). Neither former HT use nor recent HT use (i.e., current use at baseline) was associated with risk (RR, $0.98,95 \%$ CI, 0.79-1.21; RR, 0.94, 95\% CI, 0.781.13; respectively). No association was observed for duration of use ( $p$-trend $=0.46$ ) or years 
since quitting HT use ( $\leq 5$ years: RR, $1.11,95 \%$ CI, 0.80-1.55; $>5$ years: RR, $0.92,95 \%$ CI, $0.71-1.18)$. Further, we observed no variations in risk by smoking status (never, ever, former, current) (Table 2), type of HT (E-alone, E+P use), type of menopause, or lung cancer histology (data not shown).

\section{Discussion}

We observed no associations between HT-use and lung cancer risk in this large study of postmenopausal women with up to eleven years of follow-up. One explanation for the lack of effect may be the overwhelming magnitude of the association between smoking and lung cancer masking smaller associations with other exposures, such as HT. However, if this were true, we would expect to observe significant HT-use lung cancer associations among never smokers, and we did not.

A recent meta-analysis of eleven studies did not support an association between HT-use and lung cancer risk(5). In contrast, Greiser et al. reported a significant increased risk of lung cancer among never-smokers(4). However, in that study, significant associations were only found when smokers and non-smokers, various hormone regimens, or histological subtypes, respectively, were pooled(4). Furthermore, combined risk estimates from meta-analyses are difficult to interpret, in part due to study heterogeneity, and should be interpreted with considerable caution.

One limitation of our study is that residual confounding from smoking may exist. However, a major strength was our use of a five-category smoking variable that combined smoking status and median pack-years to control for both smoking status and smoking intensity.

Overall, our findings do not support an association between HT and lung cancer.

\section{Acknowledgments}

The work reported here was supported by National Cancer Institutes grants R01 CA77398, K05 CA136967, R25T CA57730, R25 056452 and R25 DA026120. The collection of cancer incidence data used in this study was supported by the California Department of Health Services as part of the statewide cancer reporting program mandated by California Health and Safety Code Section 103885; the National Cancer Institute's Surveillance, Epidemiology and End Results Program under contract N01-PC-35136 awarded to the Northern California Cancer Center, contract N01-PC-35139 awarded to the University of Southern California, and contract N02-PC-15105 awarded to the Public Health Institute; and the Centers for Disease Control and Prevention's National Program of Cancer Registries, under agreement \#U55/CCR921930-02 awarded to the Public Health Institute. The ideas and opinions expressed herein are those of the authors. Endorsement by the State of California, Department of Health Services, the National Cancer Institute, and the Centers for Disease Control and Prevention or their contractors and subcontractors is not intended nor should be inferred.

\section{References}

1. Schottenfeld, D. The etiology and epidemiology of lung cancer. In: Pass, HI.; Carbone, DP.; Johnson, DH.; Minna, JD.; Turrisi, AT., editors. Lung Cancer: Principles and Practice. 3rd ed.. Philadelphia, Pa: Lippincott Williams \& Wilkins; 2005. p. 3-24.

2. Subramanian J, Govindan R. Lung cancer in never smokers: a review. J Clin Oncol. 2007; 25:561570. [PubMed: 17290066]

3. Thomas L, Doyle LA, Edelman MJ. Lung cancer in women: emerging differences in epidemiology, biology, and therapy. Chest. 2005; 128:370-381. [PubMed: 16002959]

4. Greiser CM, Greiser EM, Doren M. Menopausal hormone therapy and risk of lung cancerSystematic review and meta-analysis. Maturitas. 2010; 65:198-204. [PubMed: 20031346]

5. Oh SW, Myung SK, Park JY, Lym YL, Ju W. Hormone therapy and risk of lung cancer: a metaanalysis. J Womens Health (Larchmt). 2010; 19:279-288. [PubMed: 20095904] 
6. Bernstein L, Allen M, Anton-Culver H, et al. High breast cancer incidence rates among California teachers: results from the California Teachers Study (United States). Cancer Causes Control. 2002; 13:625-635. [PubMed: 12296510] 
Table 1

Age-adjusted baseline characteristics among 60,592 postmenopausal California Teachers Study participants by baseline status of hormone therapy (HT) use

\begin{tabular}{|c|c|c|c|c|}
\hline \multirow{2}{*}{ Characteristic } & \multirow{2}{*}{$\underset{\text { (total) }}{\mathrm{N}}$} & \multicolumn{3}{|c|}{$\begin{array}{l}\text { HT use at baseline } \\
\text { (estrogen alone or estrogen plus progestin) }\end{array}$} \\
\hline & & Never HT user & Former HT user & Recent HT user \\
\hline No. participants & 60,592 & 15,405 & 9,716 & 35,471 \\
\hline No. invasive lung cancer cases & 727 & 216 & 147 & 364 \\
\hline Mean age at baseline \pm SD & & $65.6 \pm 11.4$ & $67.5 \pm 10.5$ & $59.7 \pm 9.4$ \\
\hline \multicolumn{5}{|l|}{ Race/ethnicity $(\%)^{l}$} \\
\hline White & 54,275 & 24.5 & 16.1 & 59.4 \\
\hline Other ${ }^{2}$ & 5,789 & 33.1 & 15.8 & 51.1 \\
\hline \multicolumn{5}{|l|}{ Menopausal status $(\%)^{3}$} \\
\hline Perimenopausal & 2,184 & 41.9 & 9.7 & 48.5 \\
\hline Postmenopausal, Natural menopause & 31,748 & 34.1 & 17.5 & 48.4 \\
\hline Postmenopausal, Other & 21,945 & 11.6 & 13.7 & 74.7 \\
\hline \multicolumn{5}{|l|}{ Smoking status $(\%)^{3}$} \\
\hline Never smoker & 37,138 & 25.2 & 14.5 & 60.3 \\
\hline Former smoker & 19,901 & 20.9 & 15.1 & 64.0 \\
\hline Current smoker & 3,553 & 29.7 & 15.4 & 54.9 \\
\hline Mean pack-years \pm SD & & $20.7 \pm 21.2$ & $20.3 \pm 20.8$ & $16.8 \pm 18.0$ \\
\hline Mean body mass index, $\mathrm{kg} / \mathrm{m}^{2} \pm \mathrm{SD}$ & & $25.7 \pm 5.5$ & $25.5 \pm 5.1$ & $24.9 \pm 4.8$ \\
\hline \multicolumn{5}{|l|}{ Body mass index, $\mathrm{kg} / \mathrm{m}^{2}(\%)^{3}$} \\
\hline$<25$ & 32,679 & 21.6 & 13.7 & 64.7 \\
\hline $25-29$ & 14,116 & 23.9 & 15.7 & 60.5 \\
\hline$\geq 30$ & 10,563 & 28.8 & 16.0 & 55.2 \\
\hline
\end{tabular}


Table 2

Multivariable-adjusted relative risks (RR) and 95\% confidence intervals (CI) for the association between hormone therapy (HT) use and incident invasive lung cancer among 60,592 postmenopausal California Teachers Study participants

\begin{tabular}{|c|c|c|c|c|c|c|}
\hline \multirow[b]{2}{*}{ HT Use } & \multicolumn{2}{|c|}{ Total } & \multicolumn{2}{|c|}{ Never Smokers } & \multicolumn{2}{|c|}{ Ever Smokers } \\
\hline & $\begin{array}{c}\mathbf{N} \\
\text { Total/Cases }\end{array}$ & $\begin{array}{l}\text { Adjusted } \\
\quad(95 \% \text { CI })\end{array}$ & $\begin{array}{c}\mathbf{N} \\
\text { Total/Cases }\end{array}$ & $\begin{array}{l}\text { Adjusted } 1,3 \text { RR } \\
(95 \% \mathrm{CI})\end{array}$ & $\begin{array}{c}\mathbf{N} \\
\text { Total/Cases }\end{array}$ & $\underset{(95 \% \text { CI })}{\text { Adjusted }}$ \\
\hline \multicolumn{7}{|l|}{ Ever HT Use } \\
\hline Never HT user & $15,405 / 216$ & 1.00 & $9,912 / 55$ & 1.00 & $5,493 / 161$ & 1.00 \\
\hline $\begin{array}{l}\text { Ever HT user (Former and } \\
\text { Recent HT users) }\end{array}$ & $45,187 / 511$ & $0.95(0.80-1.13)$ & $27,226 / 129$ & $1.06(0.76-1.50)$ & $17,961 / 382$ & $0.93(0.77-1.14)$ \\
\hline \multicolumn{7}{|l|}{ Former or Recent HT Use } \\
\hline Never HT user & $15,405 / 216$ & 1.00 & $9,912 / 55$ & 1.00 & $5,493 / 161$ & 1.00 \\
\hline Former $\mathrm{E}$ alone or $\mathrm{E}+\mathrm{P}$ user & $9,716 / 147$ & $0.98(0.79-1.21)$ & $5,871 / 43$ & $1.21(0.80-1.82)$ & $3,845 / 104$ & $0.93(0.72-1.19)$ \\
\hline Recent $\mathrm{E}$ alone or $\mathrm{E}+\mathrm{P}$ user & $35,471 / 364$ & $0.94(0.78-1.13)$ & $21,355 / 86$ & $0.98(0.67-1.43)$ & $14,116 / 278$ & $0.94(0.76-1.16)$ \\
\hline \multicolumn{7}{|l|}{ Type of HT Used } \\
\hline Never HT user & $15,405 / 216$ & 1.00 & $9,912 / 55$ & 1.00 & $5,493 / 161$ & 1.00 \\
\hline Former $\mathrm{E}$ alone or $\mathrm{E}+\mathrm{P}$ user & $9,569 / 147$ & $0.99(0.79-1.22)$ & $5,871 / 43$ & $1.20(0.79-1.81)$ & $3,845 / 104$ & $0.94(0.73-1.21)$ \\
\hline Recent $\mathrm{E}$ alone therapy & $15,663 / 188$ & $0.97(0.77-1.23)$ & $9,730 / 43$ & $0.92(0.57-1.49)$ & $5,933 / 145$ & $1.00(0.76-1.31)$ \\
\hline Recent $\mathrm{E}+\mathrm{P}$ combined therapy & $19,808 / 177$ & $0.92(0.74-1.13)$ & $11,625 / 43$ & $1.02(0.66-1.59)$ & $8,183 / 133$ & $0.89(0.70-1.14)$ \\
\hline \multicolumn{7}{|l|}{ Duration of HT Use } \\
\hline Never HT user & $15,405 / 216$ & 1.00 & $9,912 / 55$ & 1.00 & $5,493 / 161$ & 1.00 \\
\hline Ever HT user, $\leq 5$ years duration & $19,670 / 167$ & $0.93(0.76-1.15)$ & $12,205 / 48$ & $1.08(0.72-1.63)$ & $7,465 / 119$ & $0.91(0.71-1.16)$ \\
\hline $\begin{array}{l}\text { Ever HT user, 6-15 years } \\
\text { duration }\end{array}$ & $13,866 / 151$ & $0.92(0.74-1.14)$ & $8,081 / 42$ & $1.18(0.76-1.82)$ & $5,785 / 109$ & $0.85(0.66-1.10)$ \\
\hline \multirow{2}{*}{$\begin{array}{l}\text { Ever HT user, } \geq 15 \text { years } \\
\text { duration }\end{array}$} & $9,122 / 151$ & $0.95(0.75-1.20)$ & $5,399 / 30$ & $0.88(0.54-1.45)$ & $3,723 / 121$ & $0.98(0.76-1.28)$ \\
\hline & $P$-trend & 0.71 & & 0.92 & & 0.71 \\
\hline \multicolumn{7}{|l|}{ Duration of HT Use } \\
\hline Never HT user & $15,405 / 216$ & 1.00 & $9,912 / 55$ & 1.00 & $5,493 / 161$ & 1.00 \\
\hline \multicolumn{7}{|l|}{ Former HT user } \\
\hline$\leq 5$ years duration & $6,278 / 97$ & $1.04(0.82-1.33)$ & $3,802 / 31$ & $1.50(0.96-2.35)$ & $2,476 / 66$ & $0.93(0.70-1.25)$ \\
\hline $6-15$ years duration & $2,038 / 31$ & $0.89(0.60-1.31)$ & $1,224 / 8$ & $0.93(0.42-2.09)$ & $814 / 23$ & $0.87(0.55-1.36)$ \\
\hline \multirow[t]{2}{*}{$\geq 15$ years duration } & $920 / 13$ & $0.78(0.44-1.39)$ & $530 / 2$ & $0.53(0.13-2.21)$ & $390 / 11$ & $0.89(0.47-1.68)$ \\
\hline & P-trend & 0.46 & & 0.99 & & 0.48 \\
\hline \multicolumn{7}{|l|}{ Recent HT user } \\
\hline$\leq 5$ years duration & $13,392 / 70$ & $0.81(0.60-1.09)$ & $8,403 / 17$ & $0.69(0.37-1.30)$ & $4,989 / 53$ & $0.86(0.61-1.20)$ \\
\hline $6-15$ years duration & $11,828 / 120 /$ & $0.92(0.72-1.17)$ & $6,857 / 34$ & $1.25(0.77-2.02)$ & $4,971 / 86$ & $0.84(0.63-1.12)$ \\
\hline \multirow[t]{2}{*}{$\geq 15$ years duration } & 8,202138 & $0.92(0.72-1.17)$ & $4,869 / 28$ & $0.93(0.55-1.57)$ & $3,333 / 110$ & $0.93(0.70-1.23)$ \\
\hline & $P$-trend & 0.56 & & 0.60 & & 0.40 \\
\hline \multicolumn{7}{|l|}{$\begin{array}{l}\text { Years Since Last HT Use for } \\
\text { Former Users }\end{array}$} \\
\hline Never HT user & $15,405 / 216$ & 1.00 & $9,912 / 55$ & 1.00 & $5,493 / 161$ & 1.00 \\
\hline $\begin{array}{l}\text { Former HT user, } \leq 5 \text { years since } \\
\text { last use }\end{array}$ & $3,746 / 47$ & $1.11(0.80-1.55)$ & $2,176 / 10$ & $1.11(0.55-2.25)$ & $1,570 / 37$ & $1.12(0.77-1.63)$ \\
\hline
\end{tabular}




\begin{tabular}{|c|c|c|c|c|c|c|}
\hline \multirow[b]{2}{*}{ HT Use } & \multicolumn{2}{|c|}{ Total } & \multicolumn{2}{|c|}{ Never Smokers } & \multicolumn{2}{|c|}{ Ever Smokers } \\
\hline & $\begin{array}{c}\mathbf{N} \\
\text { Total/Cases }\end{array}$ & $\begin{array}{l}\text { Adjusted } 1,2 \text { RR } \\
(95 \% \mathrm{CI})\end{array}$ & $\begin{array}{c}\mathbf{N} \\
\text { Total/Cases }\end{array}$ & $\begin{array}{l}\text { Adjusted } 1,3 \text { RR } \\
(95 \% \mathrm{CI})\end{array}$ & $\begin{array}{c}\mathbf{N} \\
\text { Total/Cases }\end{array}$ & $\begin{array}{l}\text { Adjusted } 1,4 \text { RR } \\
\quad(95 \% \mathrm{CI})\end{array}$ \\
\hline $\begin{array}{l}\text { Former HT user, > } 5 \text { years since } \\
\text { last use }\end{array}$ & $5,927 / 100$ & $0.92(0.71-1.18)$ & $3,659 / 33$ & $1.32(0.83-2.11)$ & $2,268 / 67$ & $0.81(0.60-1.10)$ \\
\hline \multicolumn{7}{|l|}{${ }^{1}$ Stratified by age at baseline } \\
\hline \multicolumn{7}{|c|}{$\begin{array}{l}{ }^{2} \text { Adjusted for race, smoking status/pack-years combination (never, former light, former heavy, current light, current heavy), type of menopause } \\
\text { and BMI }\end{array}$} \\
\hline \multicolumn{7}{|c|}{${ }^{3}$ Adjusted for race, type of menopause and BMI } \\
\hline
\end{tabular}

